\title{
Potencial de resistencia a virus en especies silvestres de Solanum*
}

\author{
Gilberto Rodríguez **; Carlos Ochoa***; Luis F. Salazar***
}

\section{RESUMEN}

Se evaluó la reacción de plántulas de 67 entradas de especies silvestres de Solanum a los virus: enrollamiento de la hoja (PLRV), virus Y de la Papa (PVY), virus X de la Papa (PVX), virus S de la Papa (PVS), virus Latente de los Andes (APLV), virus Moteado Andino (APMV) y al viroide del Tubérculo Ahusado (PSTVd). Se probaron: 33 entradas de Solanum acaule, 9 de $S$. bukasovii, 5 de $S$. colombianum, 2 de $S$. chomatofilum, 1 de $S$. coelestispetalum, 2 de $S$. marinasense, 2 de $S$. raphanifolium, 1 de $S$. tundalomense y 12 entradas no identificadas. Los estudios se realizaron entre 1980-1984 en las instalaciones del Centro Internacional de la Papa (CIP), La Molina, Lima-Perú. Por las reacciones observadas se establecieron tres categorías o tipos de reacción: susceptibilidad, tolerancia y alta resistencia. $S$. acaule resultó ser altamente resistente para PLRV, PVY (inoculación mecánica de jugo infeccioso), PVX y PVS. Para APLV, la entrada OCH13389 (espécimen no identificado) se mostró con alta resistencia.

Palabras Claves Adicionales: $\quad$ Virus, resistencia, especies silvestres, Solanum.

\section{SUMMARY}

\section{Potential of Virus Resistance in Solanum Wild Species}

The reaction of seedlings of 67 accessions of wild potato species against the viruses PLRV, PVY, PVX, PVS, APLV, APMV and the viroide PSTVd was

Aceptado para publicación: Agosto 21, 1990

\footnotetext{
* $\quad$ Parte del trabajo de tesis presentado por el primer autor para optar el grado de Magister Scientiae, Escuela de Post Grado, Universidad Nacional Agraria La Molina. Apartado 456, Lima, Perú.

** Ing. Agr, M.Sc., Departamento Acad. de Fitotecnia, Facultad de Agronomía, Universidad Nacional Agraria La Molina. Apartado 456. Lima, Perú.

*** Profesor y Ph.D. respectivamente. Centro Internacional de la Papa. Apartado 5969 Lima, Perú.
} 
studied. Thirty three accessions of S. acaule, 9 of $S$. bukasovii, 5 of $S$. colombianum, 2 of $S$. chomatophihtm, 1 of $S$. coelestispetalum, 2 of $S$. marinasense, 2 of S. ruphanifolium, 1 of S. lundalomense and 12 unidentified accesions were evaluated. Studies were carried out between 1980-1984 at the International Potato Center (CIP), La Molina, Lima-Peru.

Three types of genetically controlled reactions were determined: susceptibility, tolerance and a high level of resistance. $S$. acaule showed high level of resistance to PLRV, PVY (resistance to mechanical inoculation), PVX and PVS. Seedlings of the accession OCH-13389 (unidentified species), showed high level of resistance to APLV.

Additional Index Words: Virus, resistance, wild species, Solanum

Uno de los factores limitantes en la producción de papa es la ocurrencia de enfermedades, entre ellas las ocasionadas por los virus que pueden causar pérdidas en la producción de hasta $80-90 \%$. Los virus que afectan a la papa son alrededor de 33 (2), siendo los de mayor importancia económica: PLRV, PVY, PVX, PVA, PVS, PVT, APLV, APMV, además del viroide PSTVd.

Con el desarrollo de cultivares resistentes a los virus se pueden contrarrestar las pérdidas producidas por estos patógenos; para ello se deben identificar fuentes de resistencia en especies cultivadas y silvestres de papa. En el presente trabajo se evaluó la reacción de un grupo de especies silvestres de Solanum mediante la inoculación de los virus PLRV, PVY, PVX, PVS, APLV, APMV y el viroide PSTVd.

\section{MATERIALES Y MÉTODOS}

La investigación se realizó entre 1980-1984 en las instalaciones del Centro Internacional de la Papa (CIP) en La Molina, Lima-Perú. Se evaluó la reacción de las plántulas de 67 entradas de especies silvestres del género Solanum. La semilla botánica de las entradas estudiadas las proporcionó el departamento de Taxonomía del CIP, provenientes en su mayoría de plantas originales (semilla botánica producida por las plantas en el lugar de colección). La semilla botánica, tratada con ácido giberélico (1,500 ppm), se sembró en bandejas conteniendo substrato esterilizado (arena 50\%, musgo $40 \%$ y vermiculita $10 \%$ ). 
Las plántulas se transplantaron a pastillas de musgo ("Jiffy Seven") 30-35 días después de la siembra.

Las fuentes de inoculo empleadas provinieron de plantas infectadas de: Datura stramonium y papa cv. Renacimiento para PLRV, Nicotina occidentalis para PVY, N. glutinosa para PVX ("strains" PVXc y $\mathrm{PVX}_{\mathrm{HB}}$, Chenopodium quinoa para PVS, híbrido de $N$. bigelovii x $N$. clevelandii para APLV, N. clevelandii para APMV y Lycopersicon esculentum variedad Rutgers para PSTVd.

Las plántulas de las entradas evaluadas (10 plántulas por virus y por entrada) fueron inoculadas con el jugo extraído de las plantas fuentes de inoculo con aquellos virus de transmisión mecánica y mediante áfidos virulíferos para PLRV; esta labor se realizó a los 25-30 días después del transplante.

Las detecciones serológicas de los virus se realizaron mediante los métodos de ELISA (Enzime-linked immunoabsorbent assay) y LATEX, y electroforesis para detectar PSTVd.

La secuencia de inoculaciones y los tipos de reacción obtenidas se indican a continuación:

Después de un tiempo prudencial desde la primera inoculación (3 días para los virus de inoculación mecánica y 10 días para PLRV), se empezó la lectura de síntomas. Por la reacción a los diferentes virus, se obtuvieron tres grupos de entradas:

Susceptibles: Entradas donde las plántulas muestran síntomas característicos de cada virus.

Hipersensibles: Entradas con presencia de necrosis sistémica y lesiones locales necróticas.

Tolerantes o portadores asintomáticos: Grupos de entradas sin síntomas, los cuales fueron probados por serología (para virus), y electroforesis (PSTVd).

Después de realizar las pruebas, se obtuvieron un subgrupo con reacción positiva, los cuales fueron considerados como tolerantes o portadores asintomáticos y otro subgrupo de reacción negativa, considerados como resistentes o escapes a la infección en esta etapa.

Teniendo en cuenta los resultados de la primera inoculación, nuevas plántulas del subgrupo de entradas resistentes fueron nuevamente inoculadas, llevando a cabo las evaluaciones por síntomas y serología. Las entradas probadas se agruparon en las siguientes categorías: 
Susceptibles: Grupo de entradas con síntomas (posiblemente escaparon a la infección en el primer tamizado).

Hipersensibles: Grupo de entradas con lesiones locales necróticas y necrosis sistémico.

Tolerantes, portadores asintomáticos o resistentes: Grupo de entradas sin síntomas, que fueron sometidas a pruebas serológicas.

Después de realizar las pruebas, estas entradas se agruparon en un primer subgrupo con reacción positiva siendo considerados tolerantes a la infección o portadores asintomáticos; y otro subgrupo de reacción negativa, considerados resistentes.

Las entradas que resultaron ser resistentes en el segundo tamizado, se volvieron a inocular nuevamente con los virus mediante injerto. Después de 15 a 30 días (según el virus), las plantas injertadas fueron evaluadas por síntomas y serología, las que se agrupan nuevamente en las siguientes categorías:

Resistentes a la infección: Entradas que presentan síntomas después de la inoculación por injerto (estas entradas se comportaron como resistentes a la infección después de la inoculación mecánica y transmisión por áfidos en los tamizados anteriores).

Hipersensibles: Entradas que presentan necrosis sistémicas y lesiones locales necróticas.

Resistentes a la infección, portadores asintomáticos o resistentes: Las entradas sin síntomas probadas por serología que reaccionaron positivamente fueron considerados resistentes a la infección o portadores asintomáticos, y las de reacción negativa se catalogaron como altamente resistentes o inmunes.

El grupo de entradas sin infección en la prueba anterior, fueron nuevamente injertadas a plantas indicadoras infectadas de virus a fin de confirmar su resistencia o inmunidad. 


\section{RESULTADOS Y DISCUSIÓN}

La germinación de la semilla fue buena, llegando a 100\% en la mayoría de las entradas evaluadas, garantizando los requerimientos de plántulas para los tamizados.

\section{Síntomas}

Los resultados de las evaluaciones por sintomatología para los seis virus (PLRV, PVY, PVX, PVS, APLV y APMV) y el viroide PSTVd, para las plántulas susceptibles en las entradas analizadas fueron:

Para PLRV en 22 entradas: enrollamiento (LR), clorosis (CL), detención del crecimiento (S), pigmentación (PA) y caída de hojas (Ch).

Para PVY en 37 entradas por inoculación mecánica y por injerto dos entradas (en el segundo y tercer tamizado): mosaico rugoso (Mo), necrosis $(\mathrm{N})$, lesiones locales necróticas ( $\mathrm{Lln})$ y clorosis $(\mathrm{Cl})$.

Para PVX, "strain" PVXc, en 50 entradas se observaron mosaico (Mo), necrosis apical $(\mathrm{Na})$, clorosis $(\mathrm{Cl})$, caída de hojas $(\mathrm{Ch})$, y muerte $(\mathrm{M})$, (al primer tamizado cuatro entradas). Para el "strains" $\mathrm{PVX}_{\mathrm{HB}}$, sobre 37 entradas evaluadas, 22 mostraron los síntomas indicados en el primer tamizado.

Para PVS, no presentaron síntomas por lo que todas las entradas fueron evaluadas por serología (Latex y ELISA).

Para APLV, en 32 entradas (30 al primer y dos al segundo tamizado) se observaron: moteado (Mo), lesiones locales necróticas (Lln), clorosis.

Para el viroide PSTVd, todas las entradas fueron evaluadas por electroforesis por no observarse síntomas.

El grupo de entradas susceptibles, de acuerdo a los síntomas que mostraron las plántulas como reacción a la inoculación de los virus y el viroide, se resume en la Tabla 1.

\section{Serología}

Las plántulas de las entradas que no mostraron síntomas (Tabla 1), se evaluaron mediante serología (virus) y electroforesis (viroide PSTVd). Se les consideró como portadores asintomáticos o tolerantes a la infección cuando reaccionaron como positivas, $\mathrm{y}$ resistentes cuando reaccionaron negativamente (Tabla 2). 
Tabla 1: Número de entradas donde las plántulas reaccionaron con la presencia o ausencia de síntomas frente a la inoculación de los virus y viroide.

\begin{tabular}{|l|c|c|c|c|c|c|c|c|}
\cline { 2 - 9 } \multicolumn{1}{c|}{} & PLRV & PVY & PVX $^{c}$ & PVX $_{\text {HB }}$ & PVS & APLV & APMV & PSTVd \\
\hline $\begin{array}{l}\text { Con } \\
\text { síntomas }\end{array}$ & 22 & 37 & 50 & 22 & 0 & 32 & 24 & 0 \\
\hline $\begin{array}{l}\text { Sin } \\
\text { síntomas }\end{array}$ & 45 & 30 & 17 & 15 & 67 & 35 & 43 & 67 \\
\hline $\begin{array}{l}\text { Total } \\
\text { entradas }\end{array}$ & 67 & 67 & 67 & 37 & 67 & 67 & 67 & 67 \\
\hline
\end{tabular}

Tabla 2. Reacción de las entradas sin síntomas (de la Tabla 1) evaluadas mediante serología (para los virus) y electroforesis (para PSTVd).

\begin{tabular}{|l|c|c|c|c|c|c|c|c|}
\hline Reacción & PLRV & PVY & PVX $^{\mathrm{C}}$ & PVX $_{\mathrm{HB}}$ & PVS & APLV & APMV & PSTVd \\
\hline Positiva & 14 & 28 & 15 & 15 & 58 & 34 & 43 & 52 \\
\hline Negativa & 31 & 2 & 2 & 0 & 9 & 1 & 0 & 0 \\
\hline Dudosa* & 0 & 0 & 0 & 0 & 0 & 0 & 0 & 15 \\
\hline $\begin{array}{l}\text { Total } \\
\text { entradas }\end{array}$ & 45 & 30 & 17 & 15 & 67 & 35 & 43 & 67 \\
\hline
\end{tabular}

(*) Hubo dificultades para determinar si contenían o no el viroide, ya que las muestras de las entradas evaluadas por electroforesis no prestaban confianza.

En la Tabla 3 se muestra el comportamiento de las entradas evaluadas frente a la inoculación de los virus: PLRV, PVY, PVX ("strains" PVXc y PVX $\mathrm{HB}_{\mathrm{HB}}$ ), PVS, APLV y APMV y al viroide PSTVd.

Como se notará, casi el $50 \%$ de entradas evaluadas pertenecen a Solanum acaule; se explica este hecho por el énfasis puesto en evaluar esta especie por poseer características agronómicas de mucho interés, entre ellas resistencia a las enfermedades ocasionadas por el virus PVX. Además por haberse detectado resistencia a PLRV (entradas OCH-13823 y PCH-13824) en un primer lote de entradas analizadas y finalmente porque el Banco de Germoplasma del CIP podía disponer de semilla botánica de plantas originales (semillas producidas en el lugar de colección del espécimen). 
Tabla 3. Comportamiento de las entradas estudiadas frente a los virus: PLRV, PVY, PVX (PVXc y PVX HB ), PVS, APLV y APMV y al viroide PSTVd.*

\begin{tabular}{|c|c|c|c|c|c|c|c|c|c|}
\hline ENTRADA & & PLRV & PVY & $P V X_{H B}$ & PVXwa & PVS & APLV & APM & PSTVd \\
\hline OCH-7990 & acl & RRR & $s$ & $\mathrm{~s}$ & $\mathrm{~T}$ & $\mathrm{~s}$ & $\mathrm{~s}$ & $\mathrm{~T}$ & $\mathrm{~s}$ \\
\hline $\mathrm{OCH}-8612$ & acl & RRR & $\mathrm{T}$ & $\mathrm{s}$ & $\mathrm{T}$ & $\mathrm{s}$ & $\mathrm{s}$ & $\mathrm{s}$ & $\mathrm{s}$ \\
\hline OCH-10112 & acl & RRR & $s$ & $\mathrm{~s}$ & T & $\mathrm{s}$ & $\mathrm{s}$ & $\mathrm{s}$ & $\mathrm{s}$ \\
\hline $\mathrm{OCH}-11823$ & $\mathrm{acl}$ & RRR & $S$ & RRR & $T$ & $\mathrm{~s}$ & $\mathrm{~s}$ & $\mathrm{~s}$ & $\mathrm{~s}$ \\
\hline $\mathrm{OCH}-11825$ & $\mathrm{acl}$ & RRR & $S$ & RRR & $T$ & S & S & S & S \\
\hline OCH-11826 & acl & RRR & $s$ & RT & T & $\mathrm{s}$ & $\mathrm{s}$ & $\mathrm{s}$ & $\mathrm{s}$ \\
\hline $\mathrm{OCH}-11831$ & acl & RRR & RT & $\mathrm{S}$ & T & $\mathrm{s}$ & $\mathrm{s}$ & $\mathrm{s}$ & $\mathrm{s}$ \\
\hline OCH-13196 & $\mathrm{chm}$ & $\mathrm{s}$ & $T$ & $\mathrm{~s}$ & $\mathrm{~s}$ & $\mathrm{RR}$ & T & RT & $\mathrm{s}$ \\
\hline OCH-13199 & chm & $\mathrm{s}$ & $\mathrm{T}$ & $\mathrm{s}$ & $\mathrm{s}$ & $\mathrm{RR}$ & RT & $\mathrm{T}$ & $\mathrm{s}$ \\
\hline $\mathrm{OCH}-13359$ & und & $\mathrm{S}$ & S & s & S & $\mathrm{RR}$ & $T$ & $T$ & S \\
\hline OCH-13383 & und & $\mathrm{s}$ & $s$ & $\mathrm{~s}$ & $\mathrm{~s}$ & $\mathrm{~s}$ & $\mathrm{~s}$ & $\mathrm{~s}$ & $\mathrm{~s}$ \\
\hline OCH-11384 & $\mathrm{col}$ & $\mathrm{s}$ & $\mathrm{T}$ & $\mathrm{s}$ & $\mathrm{s}$ & $\mathrm{s}$ & RS & RT & $\mathrm{s}$ \\
\hline OCH-13389 & und & $\mathrm{s}$ & $\mathrm{T}$ & $\mathrm{s}$ & $\mathrm{s}$ & $\mathrm{s}$ & RRR & RS & $\mathrm{s}$ \\
\hline $\mathrm{OCH}-13394$ & tdm & $\mathrm{S}$ & $\mathrm{T}$ & S & S & S & $\mathrm{RT}$ & $\mathrm{T}$ & S \\
\hline $\mathrm{OCH}-13397$ & col & $\mathrm{s}$ & $\mathrm{s}$ & $\mathrm{s}$ & $\mathrm{s}$ & $\mathrm{S}$ & RT & RT & $\mathrm{s}$ \\
\hline $\mathrm{OCH}-13401$ & col & $\mathrm{T}$ & S & S & $\mathrm{T}$ & $\mathrm{RR}$ & $\mathrm{T}$ & $\mathrm{T}$ & S \\
\hline $\mathrm{OCH}-13404$ & col & $\mathrm{T}$ & S & S & $\mathrm{T}$ & $\mathrm{RR}$ & $\mathrm{T}$ & $\mathrm{T}$ & S \\
\hline OCH- 13405 & col & $\mathrm{T}$ & S & S & $\mathrm{T}$ & $\mathrm{s}$ & $\mathrm{T}$ & $\mathrm{RT}$ & S \\
\hline OCH- 13735 & rap & $\mathrm{s}$ & 5 & $\mathrm{~s}$ & & $\mathrm{~s}$ & $\mathrm{~s}$ & $\mathrm{~s}$ & $\mathrm{~s}$ \\
\hline $\mathrm{OCH}-13737$ & $\mathrm{mrn}$ & $\mathrm{s}$ & $\mathrm{s}$ & S & & S & S & $\mathrm{S}$ & $\mathrm{S}$ \\
\hline OCH-13738 & $\mathrm{mrn}$ & T & T & T & & $\mathrm{s}$ & $\begin{array}{ll}\mathrm{T} \\
\mathrm{s}\end{array}$ & $\begin{array}{ll}\mathrm{T} \\
\mathrm{n}\end{array}$ & $\mathrm{s}$ \\
\hline $\mathrm{OCH}-13741$ & rap & RS & $\mathrm{T}$ & $\mathrm{T}$ & & $\mathrm{RR}$ & RS & $\mathrm{T}$ & S \\
\hline OCH- 13742 & und & $\mathrm{s}$ & $\mathrm{s}$ & $\mathrm{s}$ & & $\mathrm{s}$ & $\mathrm{s}$ & $\mathrm{s}$ & $\mathrm{s}$ \\
\hline $\mathrm{OCH}-13743$ & und & T & T & T & & $\mathrm{s}$ & T & T & $\mathrm{s}$ \\
\hline $\mathrm{OCH}-13744$ & und & $\mathrm{T}$ & $\mathrm{T}$ & $\mathrm{T}$ & & S & $\mathrm{T}$ & $\mathrm{T}$ & $\mathrm{s}$ \\
\hline $\mathrm{OCH}-13746$ & und & $\mathrm{T}$ & $\mathrm{T}$ & $\mathrm{T}$ & & S & $\mathrm{T}$ & $\mathrm{T}$ & $\mathrm{S}$ \\
\hline OCH- 13780 & und & $\mathrm{s}$ & $\mathrm{s}$ & $\mathrm{s}$ & & $\mathrm{s}$ & $\mathrm{s}$ & $\mathrm{s}$ & $\mathrm{s}$ \\
\hline $\mathrm{OCH}-13798$ & buk & $\mathrm{S}$ & $\mathrm{s}$ & $\mathrm{s}$ & & S & s & $\mathrm{S}$ & S \\
\hline OCH-13799 & buk & $\mathrm{S}$ & $\mathrm{s}$ & $\mathrm{s}$ & & $\mathrm{S}$ & $\mathrm{S}$ & $\mathrm{S}$ & S \\
\hline OCH-13800 & buk & $\mathrm{S}$ & S & S & & S & S & S & S \\
\hline OCH- 13801 & cop & $\mathrm{s}$ & $\mathrm{s}$ & $\mathrm{s}$ & & $\mathrm{s}$ & $\mathrm{s}$ & $\mathrm{s}$ & $\mathrm{s}$ \\
\hline $\mathrm{OCH}-13811$ & und & $\mathrm{s}$ & $\mathrm{s}$ & $\mathrm{s}$ & & $\mathrm{s}$ & $\mathrm{s}$ & $\mathrm{s}$ & $\mathrm{s}$ \\
\hline OCH-13812 & und & T & T & T & & $\mathrm{s}$ & T & T & $\mathrm{s}$ \\
\hline OCH-13813 & und & $\mathrm{T}$ & $\mathrm{T}$ & $\mathrm{T}$ & & $\mathrm{s}$ & T & RT & $\mathrm{s}$ \\
\hline $\mathrm{OCH}-13814$ & buk & $\mathrm{S}$ & $\mathrm{R}$ & S & & S & S & $\mathrm{s}$ & $\mathrm{S}$ \\
\hline OCH-13815 & und & $\mathrm{S}$ & $\mathrm{s}$ & $\mathrm{s}$ & & $\mathrm{s}$ & $\mathrm{S}$ & $\mathrm{s}$ & $\mathrm{s}$ \\
\hline OCH-13817 & $\mathrm{acl}$ & RRR & $\mathrm{RT}$ & $T$ & & S & $\mathrm{T}$ & $\mathrm{T}$ & $\mathrm{S}$ \\
\hline OCH-13818 & $\mathrm{acl}$ & RRR & $\mathrm{T}$ & RT & & S & $\mathrm{T}$ & $\mathrm{T}$ & $\mathrm{S}$ \\
\hline OCH-13819 & $\mathrm{acl}$ & $\mathrm{S}$ & $\mathrm{s}$ & $\mathrm{s}$ & & $\mathrm{s}$ & $\mathrm{S}$ & $\mathrm{RT}$ & $\mathrm{s}$ \\
\hline $\mathrm{OCH}-13820$ & acl & RRR & $\mathrm{T}$ & $\mathrm{T}$ & & S & S & $\mathrm{T}$ & $S$ \\
\hline OCH-13821 & $\mathrm{acl}$ & RRR & T & RT & & $\mathrm{RR}$ & $\mathrm{T}$ & RS & $\mathrm{s}$ \\
\hline $\mathrm{OCH}-13823$ & $\mathrm{acl}$ & RRRRR & s & $\mathrm{S}$ & & $\mathrm{S}$ & $\mathrm{s}$ & $\mathrm{s}$ & $\mathrm{S}$ \\
\hline OCH- 13824 & $\mathrm{acl}$ & RRRRR & $\mathrm{s}$ & $\mathrm{s}$ & & $\mathrm{S}$ & $\mathrm{S}$ & $\mathrm{s}$ & $\mathrm{s}$ \\
\hline OCH-13832 & buk & $\mathrm{s}$ & $\mathrm{s}$ & $\mathrm{s}$ & & $\mathrm{s}$ & $\mathrm{s}$ & T & $\mathrm{s}$ \\
\hline OCH-13854 & buk & $\mathrm{T}$ & T & T & & $\mathrm{s}$ & T & T & $\mathrm{s}$ \\
\hline OCH-13855 & buk & T & T & T & & $\mathrm{s}$ & $\mathrm{T}$ & RT & $\mathrm{s}$ \\
\hline OCH-13858 & buk & T & $\mathrm{T}$ & RS & & $\mathrm{s}$ & T & T & $\mathrm{s}$ \\
\hline OCH- 13860 & buk & T & T & T & & $\mathrm{s}$ & T & T & $\mathrm{s}$ \\
\hline OCH- 14267 & $\mathrm{acl}$ & RRR & $\mathrm{T}$ & S & $\mathrm{T}$ & S & S & $\mathrm{S}$ & $\mathrm{S}$ \\
\hline OCH- 14274 & $\mathrm{acl}$ & RRR & S & S & S & S & $\mathrm{S}$ & RT & $\mathrm{S}$ \\
\hline OCH- 14276 & acl & RRR & T & T & T & $\mathrm{s}$ & $\mathrm{s}$ & $\mathrm{s}$ & $\mathrm{s}$ \\
\hline OCH-14279 & $\mathrm{acl}$ & RRR & S & $\mathrm{S}$ & S & S & S & $\mathrm{S}$ & S \\
\hline OCH- 14282 & $\mathrm{acl}$ & RRR & $\mathrm{s}$ & $\mathrm{S}$ & $\mathrm{s}$ & $\mathrm{s}$ & S & $\mathrm{s}$ & - \\
\hline OCH- 14286 & $\mathrm{acl}$ & $\mathrm{T}$ & T & $\mathrm{s}$ & $\mathrm{s}$ & $\mathrm{s}$ & $\mathrm{s}$ & $\mathrm{T}$ & - \\
\hline OCH-14294 & $\mathrm{acl}$ & RRR & $\mathrm{s}$ & $\mathrm{s}$ & $\mathrm{s}$ & $\mathrm{s}$ & T & T & - \\
\hline
\end{tabular}




\begin{tabular}{|c|c|c|c|c|c|c|c|c|c|}
\hline OCH-14295 & acl & RRR & $\mathrm{S}$ & $\mathrm{S}$ & $\mathrm{S}$ & $\mathrm{S}$ & $\mathrm{T}$ & RT & - \\
\hline OCH- 14304 & $\mathrm{acl}$ & RRR & $\mathrm{S}$ & $\mathrm{S}$ & $\mathrm{S}$ & $\mathrm{S}$ & $\mathrm{T}$ & $\mathrm{T}$ & - \\
\hline OCH-14305 & $\mathrm{acl}$ & RRR & $\mathrm{S}$ & $\mathrm{S}$ & $\mathrm{S}$ & $\mathrm{S}$ & $\mathrm{T}$ & $\mathrm{T}$ & - \\
\hline $\mathrm{OCH}-14315$ & $\mathrm{acl}$ & RRR & $\mathrm{S}$ & $\mathrm{S}$ & $\mathrm{S}$ & $\mathrm{S}$ & $\mathrm{T}$ & $\mathrm{T}$ & - \\
\hline $\mathrm{OCH}-14390$ & $\mathrm{acl}$ & RRR & $\mathrm{T}$ & $\mathrm{S}$ & $\mathrm{S}$ & $\mathrm{S}$ & $\mathrm{T}$ & $\mathrm{T}$ & - \\
\hline OCH-14391 & acll & RRR & $\mathrm{S}$ & $\mathrm{S}$ & RT & $\mathrm{S}$ & RT & $\mathrm{T}$ & - \\
\hline OCH- 14392 & acl & RRR & $\mathrm{S}$ & $\mathrm{S}$ & $\mathrm{T}$ & $\mathrm{S}$ & $\mathrm{T}$ & $\mathrm{T}$ & - \\
\hline OCH-14394 & $\mathrm{acl}$ & RRR & $\mathrm{S}$ & $\mathrm{s}$ & $\mathrm{S}$ & $\mathrm{S}$ & $\mathrm{S}$ & $\mathrm{T}$ & - \\
\hline OCH- 14387 & $\mathrm{acl}$ & RRR & $\mathrm{S}$ & $\mathrm{s}$ & $\mathrm{S}$ & $\mathrm{RR}$ & $\mathrm{T}$ & $\mathrm{T}$ & - \\
\hline OCH- 14398 & acl & RRR & $\mathrm{S}$ & $\mathrm{S}$ & $\mathrm{S}$ & $\mathrm{S}$ & $\mathrm{T}$ & $\mathrm{T}$ & - \\
\hline $\mathrm{OCH}-14402$ & acl & RRR & $\mathrm{T}$ & $\mathrm{S}$ & $\mathrm{S}$ & $\mathrm{S}$ & $\mathrm{T}$ & $\mathrm{T}$ & - \\
\hline OCH- 14403 & $\mathrm{acl}$ & RRR & $\mathrm{T}$ & $\mathrm{S}$ & $\mathrm{T}$ & $\mathrm{RR}$ & RT & $\mathrm{T}$ & - \\
\hline
\end{tabular}

*El número de veces que aparece un símbolo, indica el número de veces que se repitió la prueba en dicha accesión y el resultado obtenido.

$\mathrm{S}=$ Susceptible; $\mathrm{T}=$ Tolerante o portador asintomático;

$\mathrm{R}$ = Resistente; - = Resultados de electroforesis dudosos

\begin{tabular}{llllll} 
Simbología & & & & \\
acl & $=$ & S. acaule & $c h m$ & & S chomatophilum \\
buk & $=$ & S. bukasovii & $m r n$ & $=$ & S. marinasense \\
Col & $=$ & S. colombianum & tdm & & S. tundalomense \\
cop & $=$ & S. coelestipetalum & und & & No identificadas \\
\hline
\end{tabular}

De acuerdo a los resultados, se definen tres tipos de respuesta genética de las entradas evaluadas, frente a la inoculación de los virus y del viroide PSTVd: susceptibilidad, portadores asintomáticos o tolerancia a la infección y alta resistencia. En cierto modo definiciones arbitrarias pero con aplicación a estudios similares. Estos resultados, sin embargo, son coincidentes con la clasificación de resistencia genética halladas en la literatura consultada $(1,2,4,5,7,9)$.

Susceptibilidad: definida como la capacidad de soportar la infección por las plántulas después de la inoculación del virus, desarrollando síntomas característicos de los virus inoculados en el presente trabajo. En la Tabla 1 se resumen el número de entradas que se comportaron como susceptibles y en la Tabla 3, éstos se simbolizan con la letra S. Es de notar que para el PVS y el viroide PSTVd, a pesar de no presentar síntomas se consideran como "susceptibles" a las entradas que fueron detectadas por serología (PVS) y electroforesis (PSTVd) porque generalmente son asintomáticas y difícilmente se observan síntomas.

Del grupo de entradas sin síntomas (Tabla 1), evaluadas por serología (para los virus) y electroforesis (para el viroide), se obtuvieron dos subgrupos de entradas; un grupo con reacción positiva (contenían el patógeno), a las que se denominaron portadores asintomáticos o tolerantes a la infección y otro subgrupo de reacción negativa (no contenían el patógeno), a las que se denominaron resistentes (Tabla 2). En la Tabla 3 se identifican con la letra T al primer subgrupo y con la letra $\mathrm{R}$ a las entradas del segundo subgrupo. 
El número de veces que se repite una letra o cuando le sigue otra letra (Tabla 3) significa el número de tamizados realizados para cada entrada y virus, las que a su vez han sido de ayuda para determinar el tipo de resistencia o tipo de respuesta genética.

Analizando la Tabla 3, para PLRV se detectó alta resistencia en la especie Solanum acaule en 31 entradas de 33 evaluadas; esta respuesta se obtuvo después de tres tamizados (a excepción de las entradas OCH-13823 y OCH13824 con cinco tamizados), lo cual justifica el análisis de mayor número de entradas de esta especie en el estudio. Confirmada esta característica en estudios recientes en el CIP (1989) indican que de 61 entradas probadas, $13.1 \%$ fue altamente resistente, $26.2 \%$ moderadamente resistentes y $60.7 \%$ fue susceptible (3). Para PVY se detectó resistencia a la inoculación mecánica de jugo infeccioso en dos entradas de $S$. acaule (indicadas con R y T), pero se comportaron como tolerantes a la inoculación vía injerto. Para PVX ${ }^{\mathrm{C}}$, sí se detectaron entradas resistentes (altamente resistentes) en dos entradas de $S$. acaule confirmándose una vez más esta característica determinada en otras investigaciones similares como las de Webb y Schultz en 1961 (11) quienes obtuvieron inmunidad. Ross, en 1966, determinó resistencia extrema (8) y Bagnall entre 1956-1970 (1) encontró resistencia a este virus en: S. acaule, S. kurtzianum, S. sparsipilum, S. sucrense y S. tarijense. Para PVS, se detectó resistencia en tres entradas de $S$. acaule, dos entradas de $S$. chomatophilum, dos entradas de $S$. colombianum, una entrada de $S$. raphanifolium y una entrada no identificada. Estos resultados hacen suponer que para PVS se puede contar con mayor número de especies silvestres como fuentes de resistencia sumándose a los determinados por Bagnall entre 1956-1070 (1). Para APLV, se detectaron individuos con alta resistencia en el espécimen no identificado OCH-13389, mientras que para el virus APMV y el viroide PSTVd, no se detectaron entradas con resistencia.

\section{CONCLUSIONES}

Las especies silvestres de Solanum constituyen un valioso material genético como fuente de resistencia a enfermedades ocasionadas por los virus, siendo $S$. acaule la más importante.

Se determinaron tres grupos de entradas de acuerdo a su comportamiento frente a la inoculación de los virus y el viroide: susceptibles, tolerantes a la infección o portadores asintomáticos y altamente resistentes. En este estudio no fue posible definir entre tolerantes y portadores asintomáticos porque una variedad tolerante es también un portador asintomático. Al revés, un portador asintomático no es necesariamente tolerante porque la tolerancia debe demostrarse por ensayos de rendimiento.

En el presente trabajo se detectó alta resistencia para los virus: PLRV, PVX y PVS en S. acaule. 


\section{REFERENCIAS BIBLIOGRÁFICAS}

1. Bagnall, R.H. 1972. Resistance to potato viruses M, S, X and spindle tuber viroid in tuber-bearing Solanum species. Am. Potato J. 49: 342348.

2. Bokx, JA. de. 1984. Virosis de la papa y la semilla de papa. Editorial Hemisferio Sur, Buenos Aires-Argentina. 303p.

3. Centro Internacional de la Papa. 1989. Informe Anual del CIP, 1989. Lima, Perú. 215p.

4. Fribourg, C.E. 1977. Enfermedades causadas por los virus, viroide y micoplasmas. Fitopatología Agrícola Tomo I. Universidad Nacional Agraria La Molina, Lima- Perú.

5. Hooker, W.J. 1980. Compendio de Enfermedades de la Papa, Centro Internacional de la Papa (CIP), Lima-Perú. 166p.

6. Kiehr-Delhey. 1975. Resistencia a los virus M, X e Y en especies silvestres y cultivadas argentinas de papa. Revista de Investigaciones Agropecuarias. INTA. Buenos Aires, República de Argentina. Serie S, Patología Vegetal. Vol. 11(2): 33-42.

7. Montaldo, A. 1984. Cultivo y Mejoramiento de la Papa. Instituto Interamericano de Cooperación para la Agricullura. San José, Costa Rica. 676p.

8. Ross, H. 1966. The use of wild Solanum species in german potato breeding of the past and today. Am. Potato J. 43: 275-284.

9. Salazar, L.F. 1982. Manual de enfermedades virosas de la papa. Centro Internacional de la Papa (CIP). Lima, Perú. 111 p.

10. Webb, RE.; Schultz, EJ. 1961. Resistance of Solanum species to potato viruses A, X and Y. Am. Potato J. 38: 137-142. 